\title{
The efficacy of fluoropyrimidine-based adjuvant chemotherapy on biliary tract cancer after $\mathrm{R} 0$ resection
}

\author{
Young Saing Kim ${ }^{1}$, Chi-Young Jeong ${ }^{2}$, Haa-Na Song ${ }^{3}$, Tae Hyo Kim ${ }^{3}$, Hong Jun Kim³ ${ }^{3}$ Young-Joon Lee ${ }^{2}$ \\ and Soon Chan Hong ${ }^{2}$
}

\begin{abstract}
Background: The optimal treatment strategy for biliary tract cancer (BTC) after curative-intent resection remains controversial. The purpose of this study was to evaluate the efficacy of fluoropyrimidine-based adjuvant chemotherapy for BTC patients undergoing microscopically margin-negative (RO) resection.

Methods: We retrospectively analyzed the clinical data of BTC patients who underwent curative-intent RO resection. Patients were eligible if they received either fluoropyrimidine-based adjuvant chemotherapy or observation after R0 resection.

Results: A total of 153 patients were included. In the entire patient cohort, no significant differences were observed in 5 -year overall survival (OS) rates ( $48.4 \%$ vs. $39.6 \%, P=0.439)$ or 3 -year recurrence-free survival (RFS) rates ( $49.1 \%$ vs. $39.5 \%, P=0.299$ ) between patients who received fluoropyrimidine-based adjuvant chemotherapy or observation. However, for patients with stages II and III BTC, chemotherapy significantly improved 5 -year OS rate (52.4\% vs. $35.6 \%$, $P=0.002)$ and 3 -year RFS rate (55.5\% vs. $39.1 \%, P=0.021)$ compared with observation.
\end{abstract}

Conclusion: Fluoropyrimidine-based adjuvant chemotherapy may prolong the survival of patients with stages II and III BTC after R0 resection.

Keywords: Biliary tract cancer, Adjuvant chemotherapy, Fluoropyrimidine, R0 resection, Prognosis

\section{Background}

In western countries, biliary tract cancer (BTC) is a rare disease [1, 2]. However, its incidence is high in South Korea, where it accounts for $2.4 \%$ of all diagnosed cancers and is the sixth leading cause of cancer-related death [3]. Patients with BTC usually have a poor prognosis, and only $10 \%-35 \%$ of them have a chance to undergo curative-intent resection $[4,5]$. Moreover, the recurrence rate is $30 \%-50 \%$, even after microscopically margin-negative (R0) resection [6-8].

Given the high recurrence rate of BTC, adjuvant chemotherapy is usually administered in clinical practice.

\footnotetext{
${ }^{*}$ Correspondence: zoomboom@gilhospital.com

${ }^{2}$ Department of Surgery, Institute of Health Sciences, Gyeongsang National University Hospital, Gyeongsang National University School of Medicine, 79 Gangnam-ro, Jinju 660-702, South Korea

Full list of author information is available at the end of the article
}

However, its exact role has not been determined due to the lack of randomized, prospective studies. Current information on the efficacy of adjuvant chemotherapy on BTC mostly comes from single-arm or retrospective studies. Several studies compared the efficacy of adjuvant chemotherapy with that of surgery alone; however, these studies included heterogeneous patient populations in terms of tumor location, stage, and margin status, and the conflicting results made it difficult for physicians to know who will benefit from adjuvant chemotherapy [9-15]. Some studies showed that patients with high-risk diseases, such as microscopically margin-positive (R1) resection disease, lymph node-positive disease, and advanced-stage disease, were likely to benefit from adjuvant chemotherapy [14, 15]. A meta-analysis, which included 20 studies with 6712 patients, showed that patients with BTC benefited more from adjuvant 
chemotherapy or chemoradiotherapy than from radiotherapy alone, and that the greatest benefit from adjuvant therapy was observed in patients who underwent R1 resection and/or had lymph node-positive disease [4]. However, even in this meta-analysis, the efficacy of adjuvant chemotherapy for the patients who underwent R1 resection and/or had lymph node-negative disease remains unclear.

In this study, we aimed to evaluate the efficacy of adjuvant chemotherapy in patients with R0-resected BTC.

\section{Patients and methods}

\section{Patients}

We reviewed the medical records of 193 consecutive patients who underwent surgical resection for BTC between March 1999 and December 2013 at Gyeongsang National University Hospital, Jinju, South Korea. BTC was defined as tumors of the gallbladder and the intrahepatic, perihilar, and distal bile ducts, excluding the ampulla of Vater. Patients were eligible for inclusion if they received either fluoropyrimidine-based adjuvant chemotherapy or observation alone after resection. The patients who met one of the following criteria were excluded: (1) underwent R1 or macroscopically positive margin (R2) resection, (2) died of surgical complications within 3 months after resection, (3) treated with adjuvant radiotherapy or chemoradiotherapy, (4) with another primary cancer at the time of BTC diagnosis, and (5) treated with gemcitabine-based adjuvant chemotherapy. This study was approved by the Institutional Review Board of Gyeongsang National University Hospital.

\section{Treatment}

Curative-intent R0 resection was performed in all patients in the present study, and the subsequent adjuvant chemotherapy plan and schedule were decided according to the clinicians' discretion. Adjuvant chemotherapy was started within 6-8 weeks after surgery. Patients in the adjuvant chemotherapy group were treated with either single fluoropyrimidine-based chemotherapy-including intravenous 5-fluorouracil (5-FU) and oral agents such as doxifluridine, uracil, and tegafur (UFT); capecitabine; and S-1-or combination chemotherapy consisting of 5-FU and cisplatin. Intravenous chemotherapy consisted of 5 -FU $\left(450 \mathrm{mg} / \mathrm{m}^{2}\right.$ per day) and leucovorin $\left(20 \mathrm{mg} / \mathrm{m}^{2}\right.$ per day) for 5 days every 4 weeks for 6 cycles, or 5 -FU $\left(1000 \mathrm{mg} / \mathrm{m}^{2}\right.$ on days $\left.1-4\right)$ and cisplatin $(60 \mathrm{mg} /$ $\mathrm{m}^{2}$ on day 1 ) every 3 weeks for 8 cycles. Oral regimens were as follows: doxifluridine, $800 \mathrm{mg} /$ day in two divided doses for 1 year; capecitabine, $1250 \mathrm{mg} / \mathrm{m}^{2}$ twice daily on days 1-14 every 3 weeks for 8 cycles; S-1, 40-60 mg twice daily according to body surface area on days 1-14 every 3 weeks for 8 cycles; or UFT, $300 \mathrm{mg} / \mathrm{m}^{2}$ per day in three divided doses for 1 year. Patients in the observation group were followed up after surgery without adjuvant chemotherapy or radiotherapy. Regular assessments were performed in each group using comprehensive physical examinations, tumor marker analysis, and computed tomography to detect recurrence in each group.

\section{Clinical data collection}

By medical chart review, data on the patient characteristics, including demographics, tumor location, histology, TNM stage based on the American Joint Committee on Cancer (AJCC) Cancer Staging Manual (7th edition), lymph nodal status, preoperative serum carbohydrate antigen 19-9 (CA 19-9) level, and chemotherapeutic agents, were collected. The cut-off value of serum CA 19-9 level was defined as $37 \mathrm{U} / \mathrm{mL}$ (the upper limit of normal range). Survival and recurrence data were also obtained from the medical records. Overall survival (OS) was defined as the time from the date of surgery to the date of death or the last follow-up visit. Recurrence-free survival (RFS) was defined as the time from the date of surgery to the date of first recurrence at any site or death. The follow-up consisted of abdominal computed tomography every 6 months during the first 3 years and yearly thereafter. If signs or symptoms indicated a possible recurrence, investigations were then done to verify whether the patient was recurrence-free. The follow-up cut-off date was January 21, 2014. Recurrences were divided into three patterns: locoregional recurrence, distant metastasis only, and both locoregional and distant recurrence. Locoregional and distant recurrences were defined as recurrent disease within and outside $20 \mathrm{~mm}$ of the resection margin or regional lymph node, respectively.

\section{Statistical analysis}

Categorical variables are presented as frequencies and percentages, and continuous variables are expressed as means \pm standard deviations. Clinical data were compared using the Chi squared test or Fisher's exact test for categorical variables and the Mann-Whitney $U$ test for continuous variables. OS and RFS were estimated using the Kaplan-Meier method and were compared using the log-rank test between two groups. All significant variables in univariate analysis were included in multivariate analysis. Multivariate analysis using the Cox proportional hazards model with entering selection method was performed to adjust for potential confounding factors. The results are presented as hazard ratios (HRs) and 95\% confidence intervals (CIs). A two-tailed $P$ value $<0.05$ was considered statistically significant. Missing data were omitted, and the remaining data were analyzed. SPSS software for Windows, version 21.0 (SPSS Inc., Chicago, IL, USA) was used for all statistical analyses. 


\section{Results}

\section{Patient characteristics}

A total of 153 patients were included in the study; of them, 89 (58.2\%) received fluoropyrimidine-based adjuvant chemotherapy, and $64(41.8 \%)$ were observed after surgery. Forty patients were excluded for the following reasons: $\mathrm{R} 1$ or $\mathrm{R} 2$ resection $(n=23)$; early death due to surgical complication $(n=7)$; treatment with adjuvant radiotherapy or chemoradiotherapy $(n=4)$; another primary cancer $(n=4)$; and gemcitabine-based adjuvant chemotherapy $(n=2)$. Characteristics of patients in the adjuvant chemotherapy and observation groups are shown in Table 1. Although there were more men in the observation group, no significant differences were observed in age, tumor location, histologic grade, TNM stage, lymph node status, or preoperative serum level of CA 19-9 between two groups. Most tumors were located in the gallbladder $(77 / 153,50.3 \%)$ and intrahepatic bile duct $(51 / 153,33.3 \%)$. In the adjuvant chemotherapy group, doxifluridine was most commonly used (74/89, 83.1\%), followed by 5 -FU with leucovorin $(6 / 89$, 6.7\%), 5-FU with cisplatin (4/89, 4.5\%), S-1 (3/89, 3.4\%), capecitabine $(1 / 89,1.1 \%)$, and UFT $(1 / 89,1.1 \%)$. Sixtythree of the $153(41.2 \%)$ patients experienced a recurrence. The patterns of recurrence and the proportions of patients who received additional cancer treatment after recurrence were not significantly different between two groups (Table 2).

\section{Survival analysis}

With a median follow-up of 61.2 months (range 1.1178.2 months), 46 patients in the adjuvant chemotherapy group and 20 patients in the observation group died of disease-related causes. In the entire patient cohort, 5 -year OS rates $(48.4 \%$ vs. $39.6 \%, P=0.439)$ and 3 -year RFS rates $(49.1 \%$ vs. $39.5 \%, P=0.299)$ did not differ significantly between the two groups.

To assess the relationship between tumor burden and the efficacy of fluoropyrimidine-based adjuvant chemotherapy, we compared the outcomes of patients with various primary TNM stage diseases between the adjuvant chemotherapy and observation groups. In patients with stages I and IV disease, the adjuvant chemotherapy group did not show significant differences in 5-year OS rate compared with the observation group $(P=0.612$ and $P=0.161$, respectively); whereas in patients with stages II and III diseases, the adjuvant chemotherapy group had a higher 5-year OS rate than the observation group (52.4\% vs. $35.6 \%, P=0.002$; Fig. 1a). The benefit of chemotherapy was similar in terms of RFS. In patients with stages I and IV diseases, no significant difference in 3-year RFS rate was observed between the adjuvant chemotherapy and observation groups $(P=0.785$ and $P=0.116$, respectively). However, in patients with stages II and III disease, the adjuvant chemotherapy group showed higher 3 -year RFS rate than the observation group (55.5\% vs. $39.1 \%, P=0.021$; Fig. 1b).

Additionally, we analyzed the association between the efficacy of adjuvant chemotherapy and other clinical variables (Table 3). The effects of adjuvant chemotherapy appeared to differ depending on tumor locations. In patients with distal bile duct cancer, adjuvant chemotherapy was associated with prolonged OS $(P=0.014)$ and RFS $(P=0.016)$ compared with observation. In patients with gallbladder cancer, adjuvant chemotherapy tended to improve OS rate $(P=0.057)$, but not RFS rate $(P=0.148)$. On the other hand, no significant survival benefit from adjuvant chemotherapy was observed in patients with intrahepatic cholangiocarcinoma or perihilar cholangiocarcinoma. When patients were stratified by other clinical variables such as age, lymph node status, preoperative serum CA 19-9 level, and histology, no significant differences in OS and RFS rates were observed between the adjuvant chemotherapy and observation groups.

\section{Multivariate analysis for survival}

For 85 patients with stages II and III diseases, lymph node-negative disease, low serum CA 19-9 level, and fluoropyrimidine-based adjuvant chemotherapy were favorable predictors for OS and RFS in univariate analysis. In multivariate analysis, low serum CA 19-9 level and fluoropyrimidine-based adjuvant chemotherapy were independent favorable predictors for OS and RFS (Table 4).

\section{Discussion}

Positive resection margin is considered an important predictor of poor prognosis in BTC patients who undergo surgery [2, 16-18]. Previous studies reported that adjuvant therapy was beneficial to BTC patients with R1 or R2 resection $[4,9,15,19]$. Takada et al. [9] reported that the 5 -year survival rate in patients with stages II-IV gallbladder cancer was significantly higher in the adjuvant chemotherapy group treated with mitomycin $\mathrm{C}$ and 5-FU than in the surgery alone group. In the subgroup analysis, the benefit of adjuvant chemotherapy to gallbladder cancer was observed only in patients with non-curative resection, not in those with R0 resection [9]. Another recent study showed the benefits of adjuvant chemotherapy in high-risk BTC patients with high CA 19-9 level, advanced disease, lymph node involvement, and R1 resection, but not in those with R0 resection [15]. Moreover, the two studies did not stratify patients with R0 resection by specific prognostic factors; thus, it was difficult to conclude who would benefit from adjuvant chemotherapy. In our 
Table 1 Comparison of characteristics between two groups of biliary tract cancer (BTC) patients undergoing microscopically margin-negative $(\mathrm{R} 0)$ resection

\begin{tabular}{|c|c|c|c|}
\hline Characteristic & Adjuvant chemotherapy $(n=89)$ & Observation $(n=64)$ & $P$ value \\
\hline Age (years) ${ }^{a}$ & & & 0.072 \\
\hline Median (range) & $64(36-83)$ & $67(47-80)$ & \\
\hline Gender & & & 0.048 \\
\hline Male & $50(56.2)$ & $46(71.9)$ & \\
\hline Female & $39(43.8)$ & $18(28.1)$ & \\
\hline Tumor location & & & 0.480 \\
\hline Gallbladder & $43(48.3)$ & $34(53.1)$ & \\
\hline Intrahepatic bile duct & $33(37.1)$ & $18(28.1)$ & \\
\hline Perihilar bile duct & $3(3.4)$ & $5(7.8)$ & \\
\hline Distal bile duct & $10(11.2)$ & $7(10.9)$ & \\
\hline Histologic differentiation & & & 0.225 \\
\hline Well & $34(38.2)$ & $25(39.1)$ & \\
\hline Moderate & $31(34.8)$ & $30(46.9)$ & \\
\hline Poor & $17(19.1)$ & $7(10.9)$ & \\
\hline Unspecified $^{b}$ & $7(7.9)$ & $2(3.1)$ & \\
\hline AJCC stage & & & 0.152 \\
\hline । & $26(29.2)$ & $29(45.3)$ & \\
\hline$\|$ & $35(39.3)$ & $20(31.2)$ & \\
\hline III & $18(20.2)$ & $12(18.8)$ & \\
\hline $\mathrm{IVA}^{\mathrm{C}}$ & $10(11.2)$ & $3(4.7)$ & \\
\hline Lymph node involvement & & & 0.426 \\
\hline Yes & $23(25.8)$ & $13(20.3)$ & \\
\hline No & $66(74.2)$ & $51(79.7)$ & \\
\hline CA 19-9 $(U / m L)(n=126)^{a, d}$ & & & 0.328 \\
\hline Median (range) & $25.3(0.1-11,150.0)$ & $17.6(0.6-9700.0)$ & \\
\hline
\end{tabular}

AJCC American Joint Committee on Cancer (7th edition), CA 19-9 carbohydrate antigen 19-9

a Except these, other values are presented as number of patients with percentage in parentheses

${ }^{\mathrm{b}}$ Includes undifferentiated carcinoma $(n=3)$, signet ring cell carcinoma $(n=2)$, carcinosarcoma $(n=2)$, pleomorphic carcinoma $(n=1)$, and large cell neuroendocrine carcinoma $(n=1)$

c All patients had intrahepatic bile duct cancers; 12 patients had TxN1M0 disease and 1 had T4N0M0 disease

${ }^{d}$ Baseline CA 19-9 levels were available for 77 patients in the adjuvant chemotherapy group and 49 patients in the observation group

Table 2 Comparison of patterns of recurrence and post-recurrence therapies between two groups of BTC patients undergoing $\mathbf{R O}$ resection

\begin{tabular}{|c|c|c|c|}
\hline Item & Adjuvant chemotherapy $(n=41)$ & Observation $(n=22)$ & $P$ value \\
\hline Pattern of recurrence & & & 0.893 \\
\hline Locoregional & $9(22.0)$ & $5(22.7)$ & \\
\hline Distant & $21(51.2)$ & $10(45.5)$ & \\
\hline Both & $11(26.8)$ & $7(31.8)$ & \\
\hline \multicolumn{4}{|l|}{ Post-recurrence therapy } \\
\hline Patients with any post-recurrence therapy ${ }^{\mathrm{a}}$ & $23(56.1)$ & $9(40.9)$ & 0.250 \\
\hline Chemotherapy & $18(43.9)$ & $7(31.8)$ & 0.350 \\
\hline Surgery & $3(7.3)$ & $2(9.1)$ & 1.000 \\
\hline Radiotherapy & $4(7.3)$ & $1(4.5)^{b}$ & 0.650 \\
\hline Unknown & $1(2.4)$ & $1(4.5)$ & 1.000 \\
\hline
\end{tabular}

All values are presented as number of patients with percentage in parentheses

a Three patients in the adjuvant chemotherapy group and two in the observation group received surgery and chemotherapy after recurrence

${ }^{b}$ This patient underwent concurrent chemoradiotherapy after recurrence 

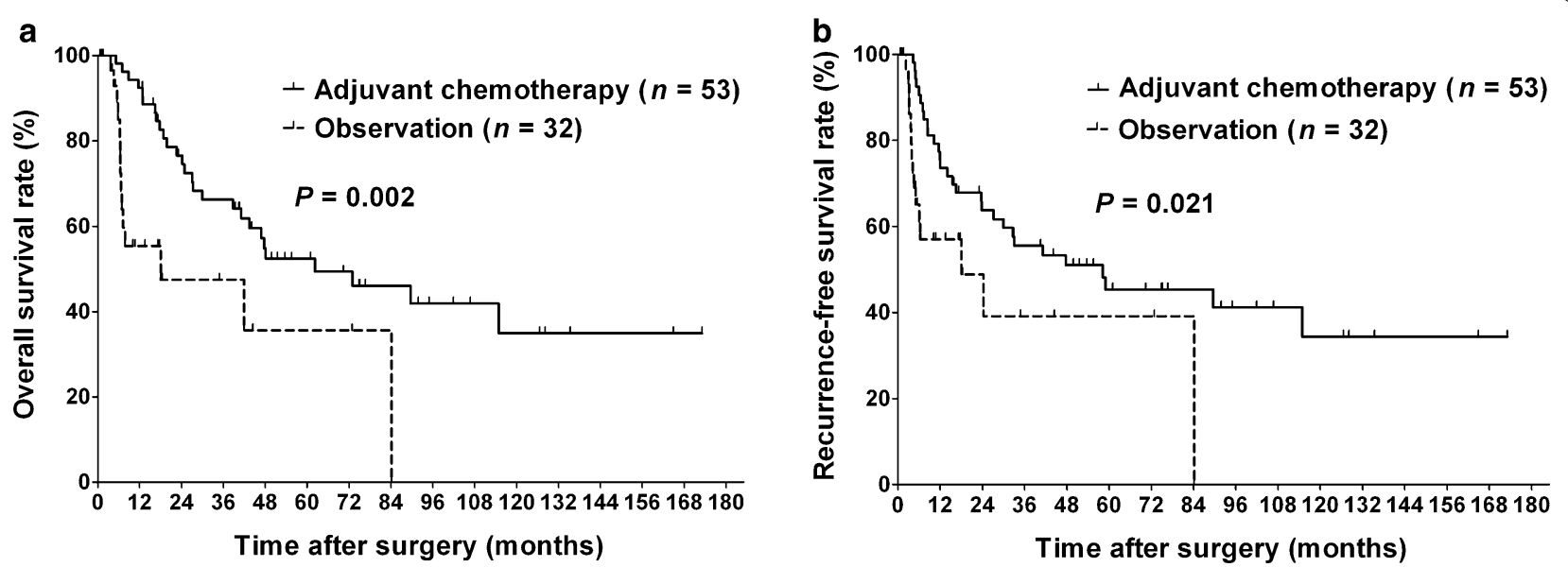

Fig. 1 Kaplan-Meier survival curves of patients with stages II and III biliary tract cancer after microscopically margin-negative resection in the fluoropyrimidine-based adjuvant chemotherapy and observation groups. a Overall survival. b Recurrence-free survival

study, we investigated the efficacy of fluoropyrimidinebased adjuvant chemotherapy in BTC patients with R0 resection. When the cohort was analyzed without stratification, no improved survivals were observed in the adjuvant chemotherapy group. However, after stratification, in patients with stages II and III disease, OS and RFS were extended by fluoropyrimidine-based adjuvant chemotherapy compared with observation alone. The efficacy of fluoropyrimidine-based adjuvant chemotherapy in patients with stages II and III BTC who underwent R0 resection was confirmed by multivariate analysis. Similarly, Yamanaka et al. [14] included only patients who underwent R0 resection for BTC and showed that adjuvant gemcitabine chemotherapy for BTC may be effective, particularly for patients with stage III BTC or intrahepatic bile duct cancer. Controversies of adjuvant chemotherapy for R0-resected BTC suggest that patients with other risk factors, such as poor tumor differentiation, lymphovascular invasion, perineural invasion, and lymph node involvement, need to be considered candidates for adjuvant chemotherapy after R0 resection.

Different from previous studies which showed that adjuvant therapy was associated with prolonged survival in patients with lymph node involvement $[4,15$, $20,21]$, our study showed that lymph node involvement did not identify patients who benefited from fluoropyrimidine-based adjuvant chemotherapy after R0 resection. Lymph node involvement indicates stages IIIB to IV BTC according to the AJCC Cancer Staging Manual (7th edition), with the exception of distal bile duct cancer. Since patients with stage IV disease in our study did not benefit from fluoropyrimidine-based adjuvant chemotherapy, lymph node involvement might not be a stratification factor to identify those who will benefit from fluoropyrimidine-based adjuvant chemotherapy. There are several possible reasons why no survival benefit from adjuvant chemotherapy was observed in patients with stage IV disease. First, the number of patients with stage IV disease $(n=13)$ was too small to have a significant difference in survival. Second, the chemotherapies in our study were limited to fluoropyrimidine-based regimens. Given that combination chemotherapy of gemcitabine and cisplatin is considered standard care for patients with advanced BTC [22], fluoropyrimidine-based adjuvant chemotherapy might not be the optimal treatment of patients with stage IV disease, even after R0 resection. Several studies evaluated gemcitabine-based adjuvant chemotherapy. Two Japanese studies showed that, as adjuvant chemotherapy, gemcitabine alone [14] or in combination with S-1 [11] prolonged OS compared with observation in the control group in patients with BTC. Neoptolemos et al. [13] found no difference in survival between adjuvant gemcitabine and fluorouracil plus leucovorin in patients with BTC. However, unlike our study, these studies of gemcitabine-based adjuvant chemotherapy included patients with ampulla of Vater cancer [11, $13,14]$, which might have different biologic characteristics compared with BTC [23] or with R1-resected BTC $[11,13]$. Comparisons between previous studies and our study are shown in Table 5. Further studies are needed to determine which regimen of adjuvant chemotherapy is more effective for BTC patients with R0 resection.

Biliary tract cancer is a heterogeneous disease that is anatomically subdivided. Resectability and surgical management vary depending on the tumor location. Each subtype is considered to have different tumor biology and prognosis [24]. In BTC patients receiving adjuvant therapy, it is also debatable which location of primary 
Table 3 Subgroup analysis of survival between two groups of BTC patients undergoing R0 resection

\begin{tabular}{|c|c|c|c|c|c|c|}
\hline \multirow[t]{2}{*}{ Variable } & \multicolumn{2}{|l|}{ 5-year OS rate (\%) } & \multirow[t]{2}{*}{$P$ value } & \multicolumn{2}{|l|}{ 3-year RFS rate (\%) } & \multirow[t]{2}{*}{$P$ value } \\
\hline & Adjuvant chemotherapy & Observation & & Adjuvant chemotherapy & Observation & \\
\hline \multicolumn{7}{|l|}{ Tumor location } \\
\hline Gall bladder & 62.5 & 52.3 & 0.057 & 69.7 & 58.1 & 0.148 \\
\hline Bile duct & 35.2 & 26.9 & 0.577 & 30.8 & 12.5 & 0.720 \\
\hline Intrahepatic bile duct & 27.5 & 0 & 0.265 & 22.8 & 32.5 & 0.549 \\
\hline Distal bile duct & 72.9 & 50.0 & 0.014 & 70.0 & 25.0 & 0.016 \\
\hline Perihilar bile duct & 0 & 66.7 & 0.139 & 0 & 33.3 & 0.066 \\
\hline \multicolumn{7}{|l|}{ Age (years) } \\
\hline$<65$ & 50.3 & 62.1 & 0.723 & 49.4 & 25.3 & 0.479 \\
\hline$\geq 65$ & 45.8 & 25.2 & 0.570 & 48.6 & 41.2 & 0.508 \\
\hline \multicolumn{7}{|l|}{ Lymph node involvement } \\
\hline Yes & 23.0 & 23.8 & 0.121 & 21.7 & 15.6 & 0.222 \\
\hline No & 57.9 & 39.6 & 0.587 & 59.3 & 44.2 & 0.336 \\
\hline \multicolumn{7}{|l|}{ CA 19-9 level $(\mathrm{U} / \mathrm{mL})^{\mathrm{a}}$} \\
\hline$<37$ & 58.7 & 75.6 & 0.195 & 62.1 & 76.7 & 0.430 \\
\hline$\geq 37$ & 34.8 & 27.9 & 0.196 & 32.3 & 0 & 0.158 \\
\hline \multicolumn{7}{|c|}{ Histologic differentiation ${ }^{b}$} \\
\hline W/D & 71.3 & 73.5 & 0.741 & 68.9 & 60.3 & 0.519 \\
\hline$M / D$ or $P / D$ & 36.6 & 29.0 & 0.476 & 39.7 & 24.9 & 0.325 \\
\hline
\end{tabular}

OS overall survival, $R F S$ recurrence-free survival, $C A$ 19-9 carbohydrate antigen $19-9, W / D$ well-differentiated, $M / D$ moderately differentiated, $P / D$ poorly differentiated

a Baseline CA 19-9 levels were unavailable for 12 patients in the adjuvant chemotherapy group and 15 patients in the observation group

b Seven patients in the adjuvant chemotherapy group and 2 patients in the observation group had unspecified carcinoma

Table 4 Multivariate analysis of survival in patients with stages II and III BTC

\begin{tabular}{llll}
\hline Prognostic factor & Hazard ratio & $\mathbf{9 5 \%} \mathrm{Cl}$ & \\
\hline Overall survival & & & \\
Lymph node (negative vs. positive) & 0.461 & $0.208-1.021$ & 0.056 \\
Serum CA 19-9 level (<37 vs. $\geq 37 \mathrm{U} / \mathrm{mL})$ & 0.379 & $0.177-0.811$ & 0.012 \\
Treatment (adjuvant chemotherapy vs. observation) & 0.231 & $0.087-0.617$ & \\
Recurrence-free survival & & 0.003 \\
Lymph node (negative vs. positive) & 0.579 & $0.269-1.250$ & $0.189-0.811$ \\
Serum CA 19-9 level (<37 vs. $\geq 37 \mathrm{U} / \mathrm{mL})$ & 0.392 & $0.161-0.923$ & 0.012 \\
Treatment (adjuvant chemotherapy vs. observation) & 0.385 & 0.032 \\
\hline
\end{tabular}

Cl confidence interval, CA 19-9 carbohydrate antigen 19-9

tumor is associated with improved survival. Jarnagin et al. [6] suggested that, based on the pattern of initial recurrence, locoregional adjuvant therapy is unlikely to have a significant effect on gallbladder cancer, whereas evidence supports its use in perihilar bile duct cancer. However, other studies showed that adjuvant chemoradiotherapy improved survival in patients with lymph node-positive gallbladder cancer $[20,21]$ or distal bile duct cancer $[25$, 26], but not in those with intrahepatic or perihilar bile duct cancer [25]. With respect to chemotherapy, as described above, benefits from adjuvant therapy were seen only in patients with gallbladder cancer [9] or intrahepatic bile duct cancer [14]. By contrast, a meta-analysis showed that there was no difference in survival between patients with gallbladder and bile duct cancers $(P=0.68)$ [4]. In our study, in patients with stages II and III distal bile duct cancer, fluoropyrimidine-based adjuvant chemotherapy was associated with significant improvement in both OS and RFS. However, this result should be interpreted with caution, considering the small number of patients $(n=17)$. Currently, it is difficult to determine which treatmentadjuvant chemotherapy or chemoradiotherapy-is more effective on the primary tumor sites in patients with BTC, especially in those undergoing $\mathrm{R} 0$ resection. 


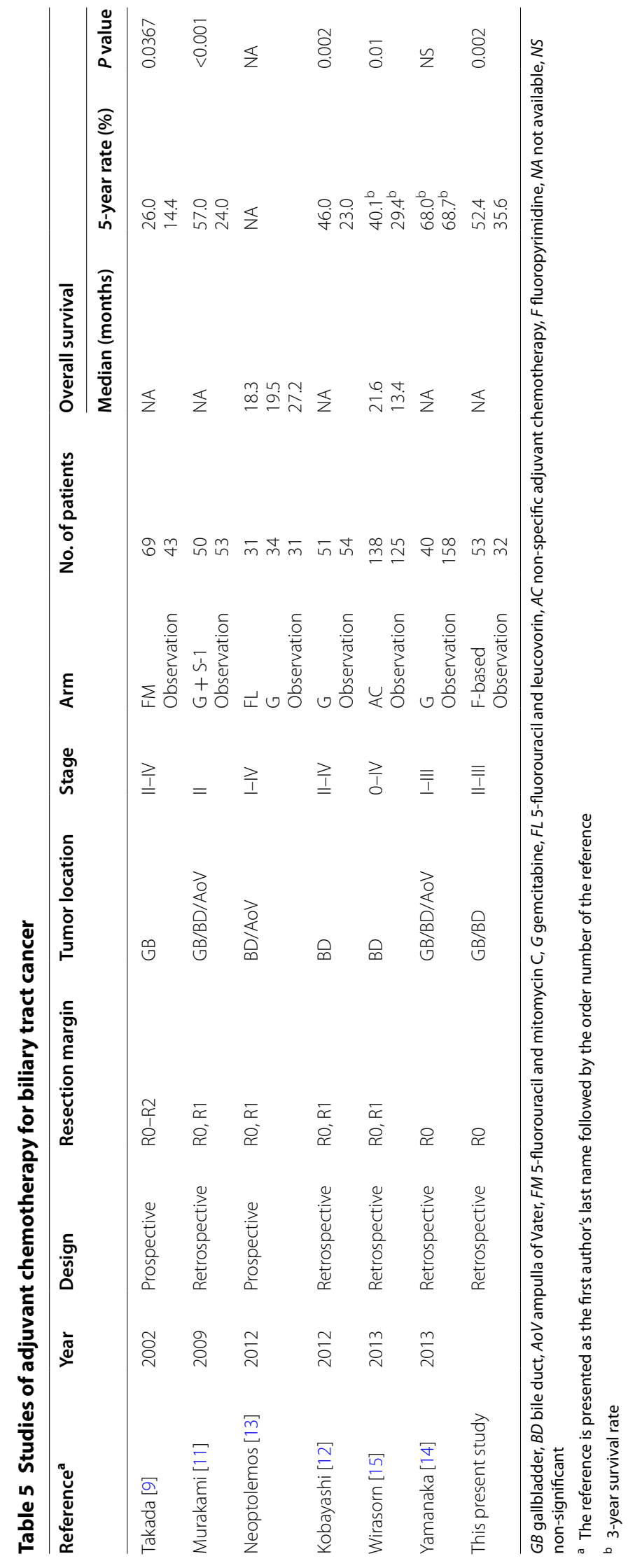


The present study had several limitations. First, this analysis was based on retrospective data from a single institution; thus, biases to single institution could not be completely avoided. Second, adjuvant chemotherapy consisted of several different chemotherapeutic regimens. Finally, the small sample size of patients with tumors in each anatomical location was insufficient to generalize our findings. Therefore, a randomized controlled trial comprising a large number of homogeneous patients with BTC is needed to confirm the efficacy of adjuvant treatment.

In conclusion, we found that fluoropyrimidine-based adjuvant chemotherapy improved survival in patients with stages II and III BTC after R0 resection. Patients with stage I disease may not benefit from adjuvant chemotherapy, and other therapeutic strategies may be considered in those with stage IV disease. In addition, the effect of adjuvant therapy appears to differ depending on the primary tumor site. Further large prospective studies are needed to validate our findings and to compare fluoropyrimidine-based adjuvant chemotherapy with other therapeutic options, such as gemcitabine-based chemotherapy and chemoradiotherapy, in BTC patients with R0 resection.

\section{Authors' contributions}

YSK and CYJ contributed to study design, literature research, and interpretation of findings. HNS collected the clinical data of patients. YSK and THK performed statistical analysis. YSK wrote the manuscript. CYJ, YJL, and SCH performed surgery and analyzed the data. HJK participated in the study design and coordination and helped draft the manuscript. All authors read and approved the final manuscript.

\section{Author details \\ ${ }^{1}$ Division of Hematology and Oncology, Department of Internal Medicine, Gachon University Gil Medical Center, Incheon 405-706, South Korea. ${ }^{2}$ Depart- ment of Surgery, Institute of Health Sciences, Gyeongsang National University Hospital, Gyeongsang National University School of Medicine, 79 Gangnam-ro, Jinju 660-702, South Korea. ${ }^{3}$ Department of Internal Medicine, Institute of Health Sciences, Gyeongsang National University Hospital, Gyeongsang National University School of Medicine, Jinju 660-702, South Korea.}

\section{Acknowledgements}

None.

\section{Competing interests}

The authors declare that they have no competing interests.

Received: 6 April 2016 Accepted: 31 December 2016

Published online: 13 January 2017

\section{References}

1. Jemal A, Siegel R, Xu J, Ward E. Cancer statistics, 2010. CA: Cancer J Clin. 2010;60(5):277-300. doi:10.3322/caac.20073.

2. Cereda S, Belli C, Reni M. Adjuvant treatment in biliary tract cancer: to treat or not to treat? World J Gastroenterol:WJG. 2012;18(21):2591-6. doi:10.3748/wjg.v18.i21.2591.

3. Jung KW, Won YJ, Kong HJ, Oh CM, Seo HG, Lee JS. Cancer statistics in Korea: incidence, mortality, survival and prevalence in 2010. Cancer Res Treat. 2013;45(1):1-14. doi:10.4143/crt.2013.45.1.1.
4. Horgan AM, Amir E, Walter T, Knox JJ. Adjuvant therapy in the treatment of biliary tract cancer: a systematic review and meta-analysis. J Clin Oncol. 2012;30(16):1934-40. doi:10.1200/JCO.2011.40.5381.

5. Zhu AX, Knox JJ. Adjuvant therapy for intrahepatic cholangiocarcinoma: the debate continues. Oncologist. 2012;17(12):1504-7. doi:10.1634/ theoncologist.2012-0432.

6. Jarnagin WR, Ruo L, Little SA, Klimstra D, D'Angelica M, DeMatteo RP, et al. Patterns of initial disease recurrence after resection of gallbladder carcinoma and hilar cholangiocarcinoma: implications for adjuvant therapeutic strategies. Cancer. 2003;98(8):1689-700. doi:10.1002/cncr.11699.

7. Li T, Qin LX, Zhou J, Sun HC, Qiu SJ, Ye QH, et al. Staging, prognostic factors and adjuvant therapy of intrahepatic cholangiocarcinoma after curative resection. Liver Int. 2014;34(6):953-60. doi:10.1111/liv.12364.

8. Kobayashi A, Miwa S, Nakata T, Miyagawa S. Disease recurrence patterns after R0 resection of hilar cholangiocarcinoma. Br J Surg. 2010;97(1):5664. doi:10.1002/bjs.6788.

9. Takada T, Amano H, Yasuda H, Nimura Y, Matsushiro T, Kato H, et al. Is postoperative adjuvant chemotherapy useful for gallbladder carcinoma? A phase III multicenter prospective randomized controlled trial in patients with resected pancreaticobiliary carcinoma. Cancer. 2002;95(8):1685-95. doi:10.1002/cncr.10831.

10. Baton O, Azoulay D, Adam DV, Castaing D. Major hepatectomy for hilar cholangiocarcinoma type 3 and 4: prognostic factors and longterm outcomes. J Am Coll Surg. 2007;204(2):250-60. doi:10.1016/j. jamcollsurg.2006.10.028.

11. Murakami Y, Uemura K, Sudo T, Hayashidani Y, Hashimoto $Y$, Nakamura $\mathrm{H}$, et al. Adjuvant gemcitabine plus S-1 chemotherapy improves survival after aggressive surgical resection for advanced biliary carcinoma. Ann Surg. 2009;250(6):950-6.

12. Kobayashi H, Murakami Y, Uemura K, Sudo T, Hashimoto Y, Kondo N, et al. Human equilibrative nucleoside transporter 1 expression predicts survival of advanced cholangiocarcinoma patients treated with gemcitabine-based adjuvant chemotherapy after surgical resection. Ann Surg. 2012;256(2):288-96. doi:10.1097/SLA.0b013e3182536a42.

13. Neoptolemos JP, Moore MJ, Cox TF, Valle JW, Palmer DH, McDonald AC, et al. Effect of adjuvant chemotherapy with fluorouracil plus folinic acid or gemcitabine vs observation on survival in patients with resected periampullary adenocarcinoma: the ESPAC-3 periampullary cancer randomized trial. JAMA. 2012;308(2):147-56. doi:10.1001/jama.2012.7352.

14. Yamanaka K, Hatano E, Kanai M, Tanaka S, Yamamoto K, Narita M, et al. A single-center analysis of the survival benefits of adjuvant gemcitabine chemotherapy for biliary tract cancer. Int J Clin Oncol. 2014;19(3):485-9. doi:10.1007/s10147-013-0578-x.

15. Wirasorn K, Ngamprasertchai T, Khuntikeo N, Pakkhem A, Ungarereevittaya P, Chindaprasirt J, et al. Adjuvant chemotherapy in resectable cholangiocarcinoma patients. J Gastroenterol Hepatol. 2013;28(12):1885-91. doi:10.1111/jgh.12321.

16. Shimada K, Sano T, Sakamoto Y, Esaki M, Kosuge T, Ojima H. Clinical impact of the surgical margin status in hepatectomy for solitary massforming type intrahepatic cholangiocarcinoma without lymph node metastases. J Surg Oncol. 2007;96(2):160-5. doi:10.1002/jso.20792.

17. Murakami Y, Uemura K, Hayashidani Y, Sudo T, Hashimoto Y, Ohge H, et al. Prognostic significance of lymph node metastasis and surgical margin status for distal cholangiocarcinoma. J Surg Oncol. 2007;95(3):207-12. doi:10.1002/jso.20668.

18. Wakai T, Shirai Y, Moroda T, Yokoyama N, Hatakeyama K. Impact of ductal resection margin status on long-term survival in patients undergoing resection for extrahepatic cholangiocarcinoma. Cancer. 2005;103(6):1210-6. doi:10.1002/cncr.20906.

19. Kim S, Kim SW, Bang YJ, Heo DS, Ha SW. Role of postoperative radiotherapy in the management of extrahepatic bile duct cancer. Int J Radiat Oncol Biol Phys. 2002;54(2):414-9.

20. Cho SY, Kim SH, Park SJ, Han SS, Kim YK, Lee KW, et al. Adjuvant chemoradiation therapy in gallbladder cancer. J Surg Oncol. 2010;102(1):87-93. doi:10.1002/jso.21544.

21. Gold DG, Miller RC, Haddock MG, Gunderson LL, Quevedo F, Donohue $\mathrm{JH}$, et al. Adjuvant therapy for gallbladder carcinoma: the Mayo Clinic Experience. Int J Radiat Oncol Biol Phys. 2009;75(1):150-5. doi:10.1016/j. ijrobp.2008.10.052.

22. Valle J, Wasan H, Palmer DH, Cunningham D, Anthoney A, Maraveyas $A$, et al. Cisplatin plus gemcitabine versus gemcitabine for 
biliary tract cancer. N Engl J Med. 2010;362(14):1273-81. doi:10.1056/ NEJMoa0908721.

23. Kim WS, Choi DW, Choi SH, Heo JS, You DD, Lee HG. Clinical significance of pathologic subtype in curatively resected ampulla of vater cancer. J Surg Oncol. 2012;105(3):266-72. doi:10.1002/jso.22090.

24. Macias RI. Cholangiocarcinoma: biology, clinical management, and pharmacological perspectives. ISRN Hepatol. 2014;2014:828074. doi:10.1155/2014/828074
25. Serafini FM, Sachs D, Bloomston M, Carey LC, Karl RC, Murr MM, et al. Location, not staging, of cholangiocarcinoma determines the role for adjuvant chemoradiation therapy. Am Surg. 2001;67(9):839-43.

26. Hughes MA, Frassica DA, Yeo CJ, Riall TS, Lillemoe KD, Cameron JL, et al. Adjuvant concurrent chemoradiation for adenocarcinoma of the distal common bile duct. Int J Radi Oncol Biol Phys. 2007;68(1):178-82. doi:10.1016/j.jijobp.2006.11.048

\section{Submit your next manuscript to BioMed Central and we will help you at every step:}

- We accept pre-submission inquiries

- Our selector tool helps you to find the most relevant journal

- We provide round the clock customer support

- Convenient online submission

- Thorough peer review

- Inclusion in PubMed and all major indexing services

- Maximum visibility for your research

Submit your manuscript at

www.biomedcentral.com/submit 